\title{
Neoadjuvant 5-fluorouracil, epirubicin and cyclophosphamide chemotherapy followed by docetaxel in refractory patients with locally advanced breast cancer
}

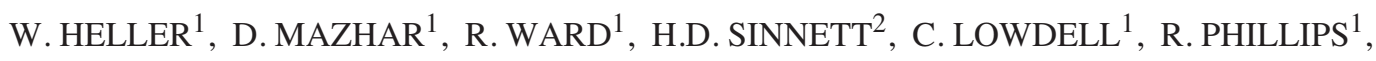 \\ S. SHOUSHA ${ }^{3}$, A. FAYAZ ${ }^{1}$, C. PALMIERI ${ }^{1}$ and R.C.COOMBES ${ }^{1}$
}

${ }^{1}$ Department of Medical Oncology, Cancer Research UK Laboratories, Faculty of Medicine, Imperial College London; Departments of ${ }^{2}$ Breast Surgery and ${ }^{3}$ Histopathology, Charing Cross Hospital, Fulham Palace Road, London W6 8RF, UK

Received July 13, 2006; Accepted October 2, 2006

\begin{abstract}
The objective of this study was to evaluate the clinical response of locally advanced breast cancer (LABC) to neoadjuvant (NA) chemotherapy with 5-fluorouracil, epirubicin and cyclophosphamide (FEC) and to study the role of docetaxel in patients who fail to respond to first-line chemotherapy. Patients were enrolled who had primary tumours without distant metastasis that were too extensive for conservative surgery. All underwent NA chemotherapy for breast cancer and thereafter surgery and/or radical radiotherapy. NA chemotherapy with FEC was administered to 88 patients between February 1998 and June 2005. A median of 6 cycles of FEC (range 1-8) was given, followed in 21 cases by a median of 4 cycles (range 2-6) of docetaxel. Where clinically established, with FEC the clinical complete response (cCR) was 22/81 (27\%), clinical partial response (cPR) 41/81 (51\%), clinical stable disease (cSD) 18/81 (22\%). In patients where the response to FEC was regarded as insufficient, docetaxel was given. Response rates were cCR 3/21 (14\%); cPR 10/21 (48\%), cSD 8/21 (38\%). There were 11 cases of pathological complete response (pCR), 9 in the FEC-only group and 2 in the docetaxel group. Following chemotherapy 49 (56\%) patients underwent mastectomy, $32(36 \%)$ breast conserving surgery and $5(6 \%)$ radical radiotherapy, giving a breast conservation rate of $42 \%$. Two patients died before receiving surgery or radical radiotherapy. The results show that neoadjuvant FEC is a reasonable NA therapy in breast cancer and that docetaxel is effective in FEC refractory cases. Only 8 of 81 (10\%) assessable patients did not respond to any chemotherapy, giving an overall clinical response rate of $90 \%$, which is comparable to studies in which taxanes were given irrespective of response to preceding therapy with antracycline including regimes.
\end{abstract}

Correspondence to: Professor R.C. Coombes, Department of Medical Oncology, Faculty of Medicine, Imperial College, Hammersmith Hospital, 6th Floor, MRC Cyclotron Building, London W12 ONN, UK

E-mail: c.coombes@imperial.ac.uk

Key words: breast cancer, neoadjuvant, FEC, docetaxel

\section{Introduction}

Neoadjuvant chemotherapy is regarded as a useful procedure in the management of locally advanced (i.e. stage III) or large ( $\geq 3 \mathrm{~cm}$ ) breast cancers. The aim of this approach is to induce tumour shrinkage in order to increase the possibility of breastconserving surgery in patients with potentially operable tumours and the response to neoadjuvant chemotherapy can be used to select subsequent adjuvant cytotoxic therapy.

Studies comparing adjuvant with neoadjuvant chemotherapy, without adjustment on the basis of response, have found higher rates of breast conserving surgery but no significant differences in terms of disease-free or overall survival have been observed (1).

Many studies have been published with various chemotherapy regimens, but we reasoned that the optimal choice of regimen would be to use as first-line the regimen we use as adjuvant therapy, i.e. the FEC regimen, and in those who fail to respond, switch to our second-line, non-cross-resistant therapy of choice, docetaxel. We felt that this approach would have two advantages: firstly, we could determine which patients were sensitive to one or other regimen, which would then be expected to inform about optimal adjuvant chemotherapy; secondly, by using the anthracycline regimen rather than combining it with docetaxel, we may expect to see less toxicity, frequently observed with the taxane-containing neoadjuvant therapy.

The chemotherapy regimes used for this approach usually include an anthracycline (doxorubicin or epirubicin) cyclophosphamide, in recent studies substituted, followed by or combined with a taxane (paclitaxel or docetaxel). The introduction of taxanes has been associated with higher response rates in most studies, but with greater toxicities (2-6; Gianni L, et al, Proc Am Soc Clin Oncol 21: abs. 132, 2002; Untch M, et al, Proc Am Soc Clin Oncol 21: abs. 133, 2002).

\section{Patients and methods}

Patient selection. This retrospective study included all patients who had received neoadjuvant chemotherapy for primary breast cancer in Charing Cross Hospital London, UK between January 
1998 and June 2005 and had then undergone surgery or radical radiotherapy without surgery.

Chemotherapy regime. Patients had received FEC every 21 days, i.e. in case of FEC 60: 5-fluorouracil $600 \mathrm{mg} / \mathrm{m}^{2}$, epirubicin $60 \mathrm{mg} / \mathrm{m}^{2}(\mathrm{n}=59)$ [50 or $75 \mathrm{mg}$ in case of FEC 50 $(n=9)$ or FEC $75(n=20)$, respectively], cyclophosphamide $600 \mathrm{mg} / \mathrm{m}^{2}$. In those patients $(\mathrm{n}=9)$ that had received FEC 50 the therapy had been repeated every 28 days with an additional dose of 5-FU and cyclophosphamide on day 8 (same dose as day 1), as described in Coombes et al (7) and Wils et al (8). In cases where the response to this therapy was not considered sufficient (i.e. no reduction in size after 2-4 cycles), we followed this by 3 -weekly docetaxel $100 \mathrm{mg} / \mathrm{m}^{2}$.

Pre-treatment evaluation. Baseline assessment included a complete medical history, physical examination, ECG, routine laboratory examinations (haematologic screen, urea, creatinine, electrolytes, liver function tests), chest X-ray, isotopic bone scan and liver sonography. Some patients had in addition CT of chest and abdomen.

The breast cancer diagnosis was confirmed by imaging (at least mammography) and histology, in the form of a core-cut biopsy. Most tumours were clinically evaluable, i.e. measurable by calliper. The tumour size was determined clinically prior to chemotherapy and on following visits to gauge the therapy response. Breast ultrasonography was repeated at the start, after 4 cycles, and at the end of treatment.

Assessment of response. The clinical response was evaluated using the Response Evaluation Criteria in Solid Tumours (RECIST) criteria by Therasse et al (9). Complete response (CR) was defined as the resolution of all target lesions; partial response (PR) was defined as at least a $30 \%$ decrease in the sum of the longest diameter of target lesions; progressive disease (PD) was defined as at least a $20 \%$ increase in the sum of the longest diameter of target lesions or the appearance of one or more new lesions; stable disease (SD) was defined as neither sufficient shrinkage to qualify for partial response nor sufficient increase to qualify for progressive disease. The same criteria have already been used in other neoadjuvant breast cancer studies by Polychronis et al (10).

A pathological complete response (pCR) was defined as no invasive tumour on histological examination (carcinoma in situ allowed) in the breast and no tumour whatsoever in the surgically removed lymph nodes.

Toxicity. Toxicity was evaluated utilising the National Cancer Institute (NCI) Common Toxicity Criteria (CTC) version 3.0 (11) (Cancer therapy evaluation program. Common Terminology Criteria for Adverse Events, Version 3.0. http://ctep. cancer.gov, December 12, 2003).

\section{Results}

Patient characteristics. We found that 88 patients had met the above criteria. They started chemotherapy between February 1998 and June 2005. The median age was 48 years with a range of 34-72 years (Table I).

The clinical tumour size prior to therapy was established precisely in 83 cases, in which the median size was $60 \mathrm{~mm}$
Table I. Pre-treatment patient and tumour characteristics.

Total no. of patients

Age range (years)

$34-72$

Median age (years)

Clinical tumour size

Established $\mathrm{n}$

Median (mm)

Range (mm)

Palpable ax. lymph nodes, $\mathrm{n}=88$

Histology

Histology available

IDC

ILC

Other invasive breast cancer

Tumour grade

Status available

1

2

3

33

(range 20-190). Five patients' tumours were difficult to measure for the following reasons: in two cases the tumour was confirmed by imaging and histology, but clinically not palpable in the breast. In one of these cases an axillary lymph node was palpable, in the other an ill-defined lesion had been confirmed by MRI. In one case the tumour was clinically described as occupying the entire breast without an initial measurement provided. In two cases no pre-treatment clinical measurement was done.

In only 2 cases the clinical tumour size was below $30 \mathrm{~mm}$. Thus the patients that had been administered neoadjuvant chemotherapy were cases of LABC as defined e.g., by Schwartz et al (12) as stage III or were at least stage IIA, regarded as worthy of consideration for NA chemotherapy (12).

Eighty-seven of 88 patients had histological results from a core biopsy; one patient had a fine-needle aspirate only. Seventy-three of $87(84 \%)$ had invasive ductal carcinoma (IDC), 11/87 (13\%) invasive lobular carcinoma (ILC), and 3/87 (3\%) other invasive breast cancer types. Oestrogen receptor status $(n=77)$ was positive in $64 \%$ of cases and $44 \%$ had grade 3 tumours. Further details are shown in Tables I and II.

Clinical response to FEC. The 88 eligible patients had received a median of 6 cycles of FEC (range 1-8). Twenty-one patients then received docetaxel chemotherapy (median 4 cycles, range 2-6) because, in 18 cases there had been no response to FEC; in 3 cases there had been a cPR to FEC, but not judged sufficient. In 2 cases the clinical response to preceding FEC had not been documented.

The clinical response rate to FEC was established in 81 of the 83 patients in whom measurements were obtained, as cCR 
Table II. Pre-treatment receptor status.

\begin{tabular}{lrr}
\hline Oestrogen receptor status & \multicolumn{2}{c}{$(\%)$} \\
Status available & 77 & $(100)$ \\
Negative & 28 & $(36)$ \\
+ & 6 & $(8)$ \\
++ & 5 & $(6)$ \\
+++ & 38 & $(50)$ \\
Progesterone receptor status & & \\
Status available & & \\
Negative & 72 & $(100)$ \\
+ & 32 & $(45)$ \\
++ & 13 & $(18)$ \\
+++ & 9 & $(12)$ \\
c-erbB-2 status & 18 & $(25)$ \\
Status available & & \\
Negative & & \\
+ & 65 & $(100)$ \\
++ & 41 & $(63)$ \\
+++ & 2 & $(3)$ \\
\hline
\end{tabular}

in $22 / 81(27 \%)$, cPR $41 / 81$ (48\%), cSD 18/81 (38\%), cPD in 0/81. A cCR was more common in patients treated with FEC $75(32 \%)$ than in patients treated with FEC $60(27 \%)$ and FEC $50(14 \%)$ (Table III).
Following neoadjuvant chemotherapy 49 patients had mastectomy, 32 patients underwent breast-conserving surgery, 5 radical radiotherapy and 2 patients died before definitive therapy. Thus the overall breast conservation rate was $42 \%$ (37/88); for FEC alone it was 45\% (30/67) (Table IV).

Clinical response to docetaxel in those failing FEC. Twentyone patients received docetaxel after FEC chemotherapy (median 4 cycles, range 2-6). And the clinical response rates were cCR 3/21 (14\%), cPR 10/21 (48\%), sSD 8/21 (38\%), cPD $0 / 21$, the breast conservation rate was $7 / 21(33 \%)$.

Pathological response. A complete pathological response occurred in 11 of $76(14 \%)$ cases where a post-operative histology (or post-chemotherapy histology in case of radical radiotherapy without surgery) was available. These tumours tended to be IDC (9/11), grade 3 (8/11), ER and c-erbB-2 negative $(6 / 9$ and $4 / 6)$.

Where histology was available before and after neoadjuvant chemotherapy, no significant trend in change of tumour characteristics was observed (histological type, grading, $\mathrm{ER} / \mathrm{PR} / \mathrm{c}$-erbB-2 status). In cases where the histological information was available (n) the tumour grade $(n=56)$ changed during neoadjuvant chemotherapy in 10 cases to a higher and in 11 cases to a lower differentiation, the ER status $(n=51)$ in 5 cases to a higher and in 6 cases to lower expression, for the PR status $(n=45)$ the corresponding figures were 6 and 10 , for the c-erbB-2 status $(n=42) 2$ and 2.

Toxicity FEC. The most common grade 3 and 4 complication was neutropenia (Table V), which occurred at least once in

Table III. Response rates.

\begin{tabular}{|c|c|c|c|c|}
\hline \multicolumn{5}{|c|}{ Response to FEC } \\
\hline \multicolumn{4}{|c|}{ No. of patients treated with FEC } & 88 \\
\hline \multicolumn{4}{|c|}{ Median of cycles FEC } & 6 \\
\hline \multicolumn{4}{|c|}{ Range of cycles FEC } & $1-8$ \\
\hline \multicolumn{4}{|c|}{ Response to FEC assessed } & 81 \\
\hline & FEC50 & FEC 60 & FEC 75 & FEC \\
\hline Response & 7 of 9 & 55 of 59 & 19 of 20 & $81-88$ \\
\hline assessed & cases $(\%)$ & cases $(\%)$ & cases $(\%)$ & cases $(\%)$ \\
\hline $\mathrm{cCR}$ & $1 \quad(14)$ & $15 \quad(27)$ & $6 \quad(32)$ & $22 \quad(27)$ \\
\hline cPR & $4 \quad(57)$ & $29 \quad(53)$ & $8 \quad(42)$ & $41 \quad(51)$ \\
\hline \multirow[t]{2}{*}{$\mathrm{cSD}$} & $2 \quad(29)$ & $11 \quad(20)$ & $5 \quad(26)$ & $18 \quad(22)$ \\
\hline & $7(100)$ & $55(100)$ & $19(100)$ & $81(100)$ \\
\hline \multicolumn{4}{|c|}{ Response to docetaxel } & $(\%)$ \\
\hline \multicolumn{4}{|c|}{ No. of patients treated with docetaxel (after FEC) } & 21 \\
\hline \multicolumn{4}{|c|}{ Median of cycles docetaxel } & 4 \\
\hline \multicolumn{4}{|c|}{ Range of cycles docetaxel } & $2-6$ \\
\hline \multicolumn{4}{|c|}{ Response to docetaxel assessed } & 21 \\
\hline \multicolumn{4}{|l|}{$\mathrm{cCR}$} & $3 / 21(14)$ \\
\hline \multicolumn{4}{|l|}{$\mathrm{cPR}$} & $10 / 21(48)$ \\
\hline \multicolumn{4}{|l|}{$\mathrm{cSD}$} & $8 / 21(38)$ \\
\hline \multicolumn{4}{|l|}{ cPD } & $0 / 21 \quad(0)$ \\
\hline
\end{tabular}


Table IV. Definitive therapy after chemotherapy.

\begin{tabular}{lcc}
\hline & $(\%)$ \\
All patients & 88 \\
Mastectomy & $49 / 88(56)$ \\
BCS & $32 / 88(36)$ \\
DXT without surgery & $5 / 88 \quad(6)$ \\
Patients RIP before definitive therapy & $2 / 88 \quad(2)$ \\
Definitive therapy after FEC & \\
only chemotherapy & \\
$\mathrm{N}$ & 67 \\
Mastectomy & $36 / 67(54)$ \\
BCS & $26 / 67(39)$ \\
DXT without surgery & $4 / 67 \quad(6)$ \\
Patient RIP before definitive therapy & $1 / 67 \quad(1)$ \\
Definitive therapy after & \\
docetaxel chemotherapy & \\
N & 21 \\
Mastectomy & $13 / 21 \quad(62)$ \\
BCS & $6 / 21(28)$ \\
Radiotherapy without surgery & $1 / 21 \quad(5)$ \\
Patient RIP before definitive therapy & $1 / 21 \quad(5)$ \\
\hline
\end{tabular}

Table V. Toxicity of chemotherapy.

\begin{tabular}{lrr}
\hline Grade 3/4 complications with FEC & \multicolumn{2}{c}{$(\%)$} \\
$\mathrm{N}$ & \multicolumn{2}{c}{88} \\
Thrombocytopenia & 0 & $(0)$ \\
Leukopenia & 16 & $(18)$ \\
Neutropenia & 30 & $(34)$ \\
Anaemia & 3 & $(3)$ \\
Nausea & 1 & $(1)$ \\
Vomiting & 1 & $(1)$ \\
Grade 3/4 complications with docetaxel & \multicolumn{2}{c}{$(\%)$} \\
$\mathrm{N}$ & \multicolumn{2}{c}{21} \\
Thrombocytopenia & 1 & $(5)$ \\
Leukopenia & 9 & $(43)$ \\
Neutropenia & 11 & $(52)$ \\
Anaemia & 2 & $(10)$ \\
Nausea & 0 & $(0)$ \\
Neutropenic fever rate & \multicolumn{2}{c}{$(\%)$} \\
FEC & $7 / 88$ & $(8)$ \\
Docetaxel & $7 / 21(33)$ \\
\hline
\end{tabular}

$34 \%$ of patients while receiving FEC. Neutropenic fever occurred at least once in 7 of the patients receiving FEC chemotherapy ( $8 \%$ for 88 patients receiving FEC). One patient died after the first cycle of FEC from a cardiac cause. Only one patient had to be admitted for grade 3/4 nausea and vomiting. Dose reductions were performed in 17 (19\%) patients during FEC. The degree of the dose reductions ranged from 10 to $50 \%$.

Toxicity docetaxel. The most common grade 3 and 4 complication was neutropenia (Table V), which occurred at least once in $52 \%$ of patients during docetaxel treatment. Neutropenic fever occurred at least once in 7 (33\%) patients. One patient died during a septic episode following docetaxel. Dose reductions were performed in $6(29 \%)$ patients during docetaxel treatment. The degree of the dose reductions ranged from 10 to $25 \%$.

\section{Discussion}

This is a retrospective study evaluating the therapeutic response to neoadjuvant chemotherapy and its translation into breast conservation in locally advanced breast cancer. The overall response rate for this study was $78 \%$ for FEC (cPR 51\% and cCR 27\%), and this translated into a breast conservation rate of $45 \%$ for patients who only received FEC. When docetaxel was given to the 21 patients where the response to initial FEC had not been regarded as sufficient, we observed a response rate of $62 \%$, translating into a breast conservation rate of $33 \%$ in these patients. The rate for pathological complete response (carcinoma in situ allowed) was $14 \%(11 / 76)$.

The baseline characteristics, such as age and median tumour size are comparable to other NA chemotherapy studies. The median age in our study was 48 years, similar to other studies, e.g. Geparduo (2), Gepartrio (3), ACCOG (13), EORTC-NCIC-SAKK (14), Aberdeen trial (4), and Diéras et al (5).

The median tumour size in this study is $60 \mathrm{~mm}$, similar to the ACCOG-trial (13). Several studies stated smaller median sizes: for example, Geparduo (2) and Gepartrio (3) quoted $40 \mathrm{~mm}$ (median), Aberdeen trial (4) $49 \mathrm{~mm}$ (median), NSABP B-18 (1) 35 mm (mean), NSABP B-27 (6) 45 mm (median). A smaller average tumour size can be assumed for a study where $>60 \%$ of tumours were T2, Diéras et al (5) as well as for the EORTC 10902 study (15) in which tumours $\leq 2 \mathrm{~cm}$ made up $14 \%$, whereas a larger average size must be supposed for the EORTC-NCIC-SAKK study (14), where $86 \%$ of the tumours were T4, as well as for Thomas et al (16), who included only $\mathrm{T} 3$ and $\mathrm{T} 4$ lesions.

The rate for overall clinical response (78\%) to FEC is comparable to other studies with anthracycline-containing regimes without a taxane. The overall clinical response rates to AC x 4 were $79 \%$ (NSABP B-18) (1), 85.5\% (NSABP B-27) (6), 70\%, Diéras et al (5), 61\% (ACCOG) (13), and $59 \%$ to CEF x 6 and $61 \%$ to EC x 6q2w (EORTC-NCICSAKK) (14), 66\% to CVAP x 8 (Aberdeen trial) (4), $83 \%$ to VACP x 3 (16), 75\% (17) and 80\% (18) to FEC $100 \times 6$. The rates for $\mathrm{CPR}$ in these studies ranged from 9 to $16 \%$.

In our study the clinical complete response rates were higher for FEC 75 (32\%) than for FEC 50 (14\%) suggesting an impact of dose intensity to the treatment outcome, but the numbers are too small to draw firm conclusions. In smaller studies clinical overall response rates for FEC 100 x 4 ranged from 64 to $72 \%$ [Servent V, et al, Breast Cancer Res Treat 94 (Suppl. 1): abs. 5074, 2005; Luporsi E, et al, Proc Am Soc 
Clin Oncol 19: abs. 355, 2000; Couteau C, et al, Proc Am Soc Clin Oncol 22: abs. 749, 2004]. For FEC 75 x 3 the clinical response rate was $62.5 \%(\mathrm{n}=16)$ (Chow LW, et al, Proc Am Soc Clin Oncol 22: abs. 327, 2003), for FEC 60 x 4 (3-weekly) a pCR of 5\% was found in a French study (Pélissier P, et al, Proc Am Soc Clin Oncol 21: abs. 254, 2002), whereas the same regime given 4-weekly for 4 cycles yielded a $10.7 \%$ pCR rate for larger tumours (median size $88 \mathrm{~mm}$ ) in a Brazilian study (Laloni MT, et al, Proc Am Soc Clin Oncol 22: abs. 832, 2004); in the same study this regime was found to be equally effective as 3-weekly AC x 4 . The quoted French study found FEC $60 \times 4$ not superior to the relatively dose intensified FEC $100 \times 4$ since they quoted similar clinical response rates (36 vs $38 \%$ ) and 5\% pCR in both groups. This is not consistent with a study that found an increased survival by using adjuvant FEC $100 \times 6$ instead of FEC 50 x 6 (19). An Indian study (20) that used the comparable but rather intensive CEF regime (cyclophosphamide $500 \mathrm{mg} / \mathrm{m}^{2}$, epirubicin $50 \mathrm{mg} / \mathrm{m}^{2}, 5$-flurouracil $500 \mathrm{mg} / \mathrm{m}^{2}$ on days 1 and 15 repeated every 4 weeks) over 3 cycles yielded a clinical overall response rate of $66 \%$, the rather low rate explainable by a high proportion of large tumours (in 46 of 50 cases tumour size over $5 \mathrm{~cm}$ ). Also the EORTC-NCIC-SAKK study had found with CEF x 6 (with oral cyclophosphamide) a clinical overall response rate of only $59 \%$ in a cohort where $86 \%$ of the tumours were T4 (14).

Even higher response rates are usually achieved with taxane-including regimes, especially if docetaxel is given subsequently to the other chemotherapy regime. Examples are the ECTO trial with APacl $\mathrm{x} 4$ followed by 4 cycles of CMF, which showed a cCR rate of $52 \%$ and a pCR in $20 \%$ (no invasive breast tumour in $23 \%$, in 87 of which lymph nodes were tumour-free), the AGO trial for Epi x 3 followed by Paclitaxel x 3q2w yielded a pCR rate of $18 \%$, Gepartrio (3) for TAC (partially followed by VCap) a pCR rate of $21.4 \%$, Diéras et al (5) a rate of overall clinical response of 89 vs $70 \%$ and a pCR rate of 16 vs $10 \%$ for APacl x 4 vs AC x 4 .

The ACCOG trial (13), however, could not demonstrate the superiority of the taxane containing regime with overall clinical response rates $\mathrm{AC} x \leq 6$ vs $\mathrm{AD} x \leq 6$ of 61 vs $70 \%$ and pCR 16 vs $12 \%$, neither could O'Regan et al (21) who compared TAC with AC. Luporsi, et al (Proc Am Soc Clin Oncol 19: abs. 355, 2000) found a similar efficacy of FEC $100 \times 4$ and ED x 4 with a pCR of $24 \%$ in both groups. In these studies docetaxel was given simultaneously with the anthracycline.

In studies where docetaxel was used sequentially after anthracyclines, the rates for overall clinical response (pathological complete response rate) were $85 \%$ (22\%, Geparduo) (2), $90.7 \%$ (26.1\%, NSABP B-27) (6), 94\% (34\%, Aberdeentrial) (4). The overall clinical response rates of 4 cycles of FEC 100 were increased to 71 and $93 \%$ by following with 4 cycles of docetaxel $100 \mathrm{mg} / \mathrm{m}^{2}$, to $71.4 \%$ by adding 4 cycles of docetaxel $75 \mathrm{mg} / \mathrm{m}^{2}$ [Ohno S, et al, Breast Cancer Res Treat 88 (Suppl. 1): abs. 2103, 2004].

The relatively low response rate for docetaxel $(62 \%)$ in our study can easily be explained by the fact that only patients with an insufficient response to the first line therapy with FEC were treated with docetaxel. Another study that also reports response rates of docetaxel for the non-anthracycline-sensitive cases is the Aberdeen trial (4), where the response rate to docetaxel in these cases was $47 \%$ (36\% cPR and $11 \% \mathrm{cCr}$ ).

A pCR, a strong indicator of long-term outcome (4), appeared more common with IDC, grade 3 tumours, ER- and c-erbB-2 negative receptor status. This is consistent with the finding that ILC shows a poor response rate to neoadjuvant chemotherapy (22-24). The tendency towards a high response rate in grade 3 and hormone receptor negative tumours has been confirmed by other trials $(2,3,5,6,18,25,26)$, whereas a trend towards a higher response rate in hormone receptor positive tumours (not statistically significant) was seen by Burcombe et al (27).

A tendency of better response of c-erbB-2 positive tumours to chemotherapy in contrast to c-erbB-2 negative tumours has been found by Learn et al (28) as well as by Penault-Llorca et al (29), whereas the opposite was stated by Gregory et al (30), Chang et al (31), and Burcombe et al (27) who found that c-erbB-2 positive patients were much less likely to respond to chemoendocrine therapy or chemotherapy, respectively; other authors found no correlation between response and c-erbB-2 status at all $(3,18,24,32,33)$.

Significant changes of the tumour biological parameters brought about by chemotherapy could not be demonstrated in our cohort. In the literature a trend towards a downgrading of tumours during chemotherapy was observed by Amat et al especially in responding tumours with a trend towards upgrading in non-responding tumours (26), a tendency towards a lowering of expression of oestrogen and progesterone receptors by Taucher et al (34). Other authors found no significant modulation of tumour characteristics $[(27,32)$ c-erbB-2, hormone receptors; (35) histological grade].

In our study the response to neoadjuvant chemotherapy translated into a breast conservation rate of $45 \%$ in the FEConly and of $33 \%$ in the FEC-docetaxel group, giving an overall breast-conservation rate of $42 \%$. Our study therefore showed a lower breast conservation rate than others. For regimes without a taxane the BCR ranges from 20\% (ACCOG) (13) to $82 \%$ (Scholl1994/ Institut Curie) (36), for regimes with a taxane from 20\% (ACCOG) (13) to 75\% (2). This discrepancy is most likely due to a lack of standardisation of the indication for mastectomy vs breast conserving therapy as e.g. discussed in the EORTC-NCIC-SAKK study (14).

We found a rather high rate of neutropenic fever or sepsis in patients treated with docetaxel of 7/21 cases in contrast to only $7 / 88$ for patients during FEC chemotherapy. Figures for the neutropenic fever/sepsis rate have been given for regimes without docetaxel as 7.3 vs $21.2 \%$ with docetaxel (NSABP B-27) (6), in the ACCOG trial (13) 12 vs $24 \%$. It is thus evident that docetaxel carries a higher risk of neutropenic fever and several studies reported cases of death with docetaxel, mostly attributable to this complication.

The Geparduo study reported 3 fatalities during chemotherapy ( 2 due to pulmonary embolism, 1 to unknown cause), the Gepartrio study 1 fatality following TAC, the NSABP B-27 (6) study 7 fatalities (3 by sepsis) related to AD in contrast to 2 with AC. The Aberdeen trial (4) reported 2 fatalities in the docetaxel group. The EORTC-NCIC-SAKK group (14) reported 2 cases of death with CEF (one due to heart failure, one due to febrile neutropenia). Antibiotic prophylaxis given 
with the first cycle of chemotherapy does not seem to influence significantly the neutropenic fever rate, since in the ACCOG (13) trial the rate of this complication was $24 \%$ with $\mathrm{AD}$ despite ciprofloxacin medication and of $14 \%(\mathrm{CEF})$ and $8.4 \%$ (ECq2w) with continuous trimethoprim-sulfomethoxazole in the EORTC-NCIC-SAKK trial (14).

We conclude that neoadjuvant chemotherapy with FEC, followed by docetaxel is a reasonable option in the treatment of locally advanced breast cancer. Despite encouraging results, the side effects of chemotherapy, especially neutropenic fever with docetaxel, have to be taken into account. Starting with the FEC regimen means that a large proportion of patients can be spared the toxicity of docetaxel. By giving the taxane only to patients that fail to respond satisfactorily to FEC, an overall clinical response of about $90 \%$ can be achieved. This rate is comparable to quoted studies in which docetaxel was given to every patient irrespective of the response to the anthracycline containing regime.

Further cross-study comparisons will be facilitated by standardising the surgical procedure following NA chemotherapy as well as the establishment of guidelines for preselecting patients for neoadjuvant chemotherapy, especially taking into account the encouraging results of studies combining chemotherapy with different classes of drugs. An example of this is the use of trastuzumab in combination with paclitaxel and FEC 75 leading to a complete pathological response rate of $65.2 \%$ (37), but also the combination with celecoxib, leading to an improvement of this rate in a small study ( $\mathrm{n}=16$ each group) from 6.3 to $12.5 \%$ by adding it to 3 cycles of FEC 75 .

\section{Acknowledgements}

RCC is supported by Cancer Research UK.

\section{References}

1. Wolmark N, Wang J, Mamounas E, et al: Preoperative chemotherapy in patients with operable breast cancer: nine-year results from National Surgical Adjuvant Breast and Bowel Project B-18. J Natl Cancer Inst Monogr 30: 96-102, 2001.

2. Von Minckwitz G, Raab G, Caputo A, et al: Doxorubicin with cyclophosphamide followed by docetaxel every 21 days compared with doxorubicin and docetaxel every 14 days as preoperative treatment in operable breast cancer: the GEPARDUO study of the German Breast Group. J Clin Oncol 23: 2676-2685, 2005.

3. Von Minckwitz G, Blohmer JU, Raab G, et al: In vivo chemosensitivity-adapted preoperative chemotherapy in patients with early-stage breast cancer: the GEPARTRIO pilot study. Ann Oncol 16: 56-63, 2005.

4. Smith IC, Heys SD, Hutcheon AW, et al: Neoadjuvant chemotherapy in breast cancer: significantly enhanced response with docetaxel. J Clin Oncol 20: 1456-1466, 2002.

5. Diéras V, Fumoleau P, Romieu G, et al: Randomized parallel study of doxorubicin plus paclitaxel and doxorubicin plus cyclophosphamide as neoadjuvant treatment of patients with breast cancer. J Clin Oncol 22: 4958-4965, 2004.

6. Bear HD, Anderson S, Brown A, et al: The effect on tumor response of adding sequential preoperative docetaxel to preoperative doxorubicin and cyclophosphamide: preliminary results from National Surgical Adjuvant Breast and Bowel Project Protocol B-27. J Clin Oncol 21: 4165-4174, 2003.

7. Coombes RC, Bliss JM, Wils J, et al: Adjuvant cyclophosphamide, methotrexate and fluorouracil versus fluorouracil, epirubicin and cyclophosphamide chemotherapy in premenopausal women with axillary node-positive operable breast cancer: results of a randomized trial. The International Collaborative Cancer Group. J Clin Oncol 14: 35-45, 1996.
8. Wils J, Coombes RC, Marty M, et al: Design and rationale of a randomised comparison of cyclophosphamide, methotrexate and fluorouracil vs fluorouracil, epirubicin and cyclophosphamide in node-positive premenopausal women with operable breast cancer. A trial of the International Collaborative Cancer Group (ICCG). Drugs 45 (Suppl. 2): S46-S50, 1993.

9. Therasse P, Arbuck SG, Eisenhauer EA, et al: New guidelines to evaluate the response to treatment in solid tumors. J Natl Cancer Inst 92: 205-216, 2000.

10. Polychronis A, Sinnett HD, Hadjiminas D, et al: Preoperative gefitinib versus gefitinib and anastrozole in post-menopausal patients with oestrogen-receptor positive and epidermal-growthfactor-receptor-positive primary breast cancer: a double-blind placebo-controlled phase II randomised trial. Lancet Oncol 6: 383-391, 2005.

11. Trotti A, Colevas AD, Setser A, et al: CTCAE v3.0: development of a comprehensive grading system for the adverse effects of cancer treatment. Semin Radiat Oncol 13: 176-181, 2003.

12. Schwartz GF, Hortobagyi GN, Masood S, et al: Proceedings of the consensus conference on neoadjuvant chemotherapy in carcinoma of the breast, April 26-28, 2003, Philadelphia, PA, USA. Hum Pathol 35: 781-784, 2004.

13. Evans TR, Yellowlees A, Foster E, et al: Phase III randomized trial of doxorubicin and docetaxel versus doxorubicin and cyclophosphamide as primary medical therapy in women with breast cancer: an anglo-celtic cooperative oncology group study. J Clin Oncol 23: 2988-2995, 2005.

14. Therasse P, Mauriac L, Welnicka-Jaskiewicz M, et al: Final results of a randomized phase III trial comparing cyclophosphamide, epirubicin, and fluorouracil with a dose-intensified epirubicin and cyclophosphamide + filgrastim as neoadjuvant treatment in locally advanced breast cancer: an EORTC-NCICSAKK multicenter study. J Clin Oncol 21: 843-850, 2003.

15. Van der Hage JA, van de Velde CJ, Julien JP, et al: Preoperative chemotherapy in primary operable breast cancer: results from the European Organization for Research and Treatment of Cancer trial 10902. J Clin Oncol 19: 4224-4237, 2001.

16. Thomas E, Holmes FA, Smith TL, et al: The use of alternate, non-cross-resistant adjuvant chemotherapy on the basis of pathologic response to a neoadjuvant doxorubicin-based regimen in women with operable breast cancer. Long-term results from a prospective randomized trial. J Clin Oncol 22: 2294-2302, 2004.

17. Mouret-Reynier MA, Abrial CJ, Ferrière JP, et al: Neoadjuvant FEC 100 for operable breast cancer: eight-year experience at Centre Jean Perrin. Clin Breast Cancer 5: 303-307, 2004.

18. Petit T, Wilt M, Velten M, et al: Comparative value of tumour grade, hormonal receptors, Ki-67, HER-2 and topoisomerase II alpha status as predictive markers in breast cancer patients treated with neoadjuvant anthracycline-based chemotherapy. Eur J Cancer 40: 205-211, 2004.

19. French Adjuvant Study Group: Benefit of a high-dose epirubicin regimen in adjuvant chemotherapy for node-positive breast cancer patients with poor prognostic factors: 5-year follow-up results of French Adjuvant Study Group 05 randomized trial. J Clinic Oncol 19: 602-611, 2001.

20. Deo SV, Bhutani M, Shukla NK, et al: Randomized trial comparing neo-adjuvant versus adjuvant chemotherapy in operable locally advanced breast cancer (T4b N0-2 M0). J Surg Oncol 84: 192-197, 2003.

21. O'Regan RM, von Roenn JH, Carlson RW, et al: Final results of a phase II trial of preoperative TAC (docetaxel/doxorubicin/ cyclophosphamide) in stage III breast cancer. Clin Breast Cancer 6: 163-168, 2005.

22. Cristofanilli M, Gonzalez-Angulo A, Sneige K, et al: Invasive lobular carcinoma classic type: response to primary chemotherapy and survival outcomes. J Clin Oncol 23: 41-48, 2005.

23. Cocquyt VF, Blondeel PN, Depypere HT, et al: Different responses to preoperative chemotherapy for invasive lobular and invasive ductal breast carcinoma. Eur J Surg Oncol 29: 361-367, 2003.

24. Pu RT, Schott AF, Sturtz DE, et al: Pathologic features of breast cancer associated with complete response to neoadjuvant chemotherapy: importance of tumor necrosis. Am J Surg Pathol 29: 354-358, 2005.

25. Kuerer HM, Sahin AA, Hunt KK, et al: Incidence and impact of documented eradication of breast cancer axillary lymph node metastases before surgery in patients treated with neoadjuvant chemotherapy. Ann Surg 230: 72-78, 1999. 
26. Amat S, Penault-Llorca F, Cure H, et al: Scarff-Bloom-Richardson (SBR) grading: a pleiotropic marker of chemosensitivity in invasive ductal breast carcinomas treated by neoadjuvant chemotherapy. Int J Oncol 20: 791-796, 2002.

27. Burcombe RJ, Makris A, Richman PI, et al: Evaluation of ER, PgR, HER-2 and Ki-67 as predictors of response to neoadjuvant anthracycline chemotherapy for operable breast cancer. Br J Cancer 92: 147-155, 2005.

28. Learn PA, Yeh IT, McNutt M, et al: HER-2/neu expression as a predictor of response to neoadjuvant docetaxel in patients with operable breast carcinoma. Cancer 103: 2252-2260, 2005.

29. Penault-Llorca F, Cayre A, Bouchet Mishellany F, et al: Induction chemotherapy for breast carcinoma: predictive markers and relation with outcome. Int J Oncol 22: 1319-1325, 2003.

30. Gregory RK, Powles TJ, Salter J, et al: Prognostic relevance of cerbB2 expression following neoadjuvant chemotherapy in patients in a randomised trial of neoadjuvant versus adjuvant chemoendocrine therapy. Breast Cancer Res Treat 59: 171-175, 2000.

31. Chang J, Powles TJ, Allred DC, et al: Biologic markers as predictors of clinical outcome from systemic therapy for primary operable breast cancer. J Clin Oncol 17: 3058-3063, 1999.

32. Schneider J, Lucas R, Sanchez J, et al: Modulation of molecular marker expression by induction chemotherapy in locally advanced breast cancer: correlation with the response to therapy and the expression of MDR1 and LRP. Anticancer Res 20: 4373-4377, 2000.
33. Bottini A, Berruti A, Bersiga A, et al: Effect of neoadjuvant chemotherapy on Ki67 labelling index, c-erbB-2 expression and steroid hormone receptor status in human breast tumours. Anticancer Res 16: 3105-3110, 1996.

34. Taucher S, Rudas M, Gnant M, et al: Sequential steroid hormone receptor measurements in primary breast cancer with and without intervening primary chemotherapy. Endocr Relat Cancer 10: 91-98, 2003.

35. Aas T, Geisler S, Helle H, et al: Prognostic and predictive value of changes in tumour cell proliferation in locally advanced breast cancer primarily treated with doxorubicin. Oncol Rep 13: 525-530, 2005.

36. Scholl SM, Fourquet A, Asselain B, et al: Neoadjuvant versus adjuvant chemotherapy in premenopausal patients with tumours considered too large for breast conserving surgery: preliminary results of a randomised trial: S6. Eur J Cancer 30A: 645-652, 1994.

37. Buzdar AU, Ibrahim NK, Francis D, et al: Significantly higher pathologic complete remission rate after neoadjuvant therapy with trastuzumab, paclitaxel and epirubicin chemotherapy: results of a randomized trial in human epidermal growth factor receptor 2-positive operable breast cancer. J Clin Oncol 23: 3676-3685, 2005. 